\title{
Material Flow in FSW of T-joints: Experimental and Numerical Analysis
}

\author{
G. Buffa ${ }^{1}$, L. Fratini ${ }^{1}$, F. Micari ${ }^{1}$, R. Shivpuri ${ }^{2}$ \\ ${ }^{1}$ Dipartimento di Tecnologia Meccanica, Produzione e Ingegneria Gestionale \\ Università di Palermo, Viale delle Scienze 90128 Palermo, Italy \\ URL:web.dtpm.unipa.it/mtge-mail:g.buffa@dtpm.unipa.it; abaqus@dtpm.unipa.it; micari@dtpm.unipa.it
}

\author{
${ }^{2}$ The Ohio State University, Department of Industrial, Welding and Systems Engineering \\ 1971 Neil Avenue, 210 Baker Systems, Columbus, Ohio 43210, USA \\ URL: iwse.osu.edu \\ e-mail: shivpuri.1@osu.edu;
}

\begin{abstract}
In the paper the authors present the results of both an experimental and a numerical campaign focused on the analysis of the occurring material flow in the FSW of T joints of aluminum alloys. In particular to investigate the metal flow experimental tests and observations has been developed utilizing a thin foil of copper as marker placed between the skin and the stringer. In this way, the actual metal flow occurring during the FSW of T-joints has been highlighted together with the real bonding surface. The acquired information is definitively useful in order to choose effective set of process parameters, improving the process mechanics and avoiding the insurgence of defects.
\end{abstract}

Key words: FSW, T-Joints, FEM, material flow.

\section{INTRODUCTION}

Friction stir welding (FSW) is an energy efficient and environmentally "friendly" (no fumes, noise, or sparks) welding process, patented in 1991 by TWI, during which the workpiece are welded together in a solid-state joining process at a temperature below the melting point of the workpiece material under a combination of extruding and forging. The process mechanics is based on the frictional heat which causes the workpiece material to soften reaching temperatures just below the melting point and allows the tool to traverse the weld line while generating plastic deformation in the workpiece; also a significant microstructural evolution takes place during FSW due and continuous dynamic recrystallization (CDRX) phenomena resulting in a highly refined grain structure in the weld nugget and permitting to obtain effective welded joints without any external heat supplier or gas protection, [1-2].

The introduction of such joining technology, due to its performance, simplifies the production scenario of particular shaped profiles. It is well known for instance that in the aircraft and aerospace industries complex profiles and joining of so-called 'skin and stringers' are very often utilized for flying structures bodies. In recent years a few results have been presented that investigate the development of welded T-joints, made of lightweight alloys, trough FSW [3-4] with encouraging results. A lack of knowledge is however still present on the mechanics of the FSW of T joints. It should be observed that the full comprehension of the process mechanics of FSW is strictly connected to the clear highlighting and understanding of the material flow occurring during the process itself. In this way a few investigations can be found out in literature starting from the developments of mixed joints in order to study the mixing of the two materials and then the occurring metal flow [5], but no research is known by the authors regarding the material flow occurring in FSW of $\mathrm{T}$ joints.

In the paper the material flow in the FSW of T joints of AA2024-T4 aluminum alloy is investigated by means of both numerical simulations and experiments. In particular to investigate the metal flow a campaign of experimental tests and observations has been developed utilizing a thin foil of copper as marker placed between the skin and the stringer. In this way, the actual metal flow occurring during the FSW of T-joints has been highlighted. 


\section{EXPRERIMENTAL AND NUMERICAL} FEATURES

\subsection{Experimental setup}

FSW of T-joints is obtained inserting a specially designed rotating pin into the clamped blanks as shown in Figure 1 and then moving it all along the joint. The pin is inserted with a proper nuting angle limiting the contact between the tool shoulder and the blank.

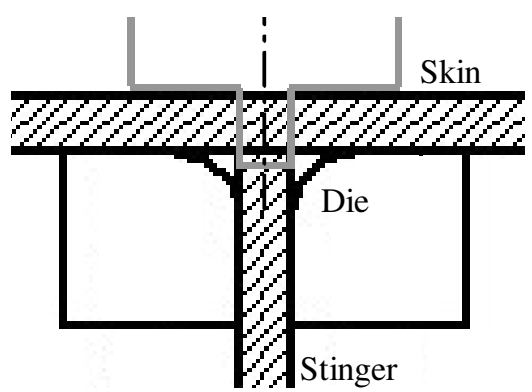

Fig. 1. Positioning of the tool and the two blanks (skin and stringer)

As the pin is inserted into the upward sheet (skin), the blank material undergoes to a local backward and forward extrusion process up to penetrate the vertical blank (stringer) and to reach the tool shoulder contact. Then the tool rotation determines an increase of the material temperature due to the friction forces work. In this way the material mechanical characteristics are locally decreased and the blanks material reaches a sort of "soft" state; no melting is observed. Then the tool can be moved along the joint. The tool movement determines heat generation due to both friction forces work and material deformation one.

In order to develop the FSW operations a properly designed clamping fixture was utilized in order to fix the specimens to be welded on a milling machine.

As far as the developed processes are regarded, $3 \mathrm{~mm}$ thick AA6082-T6 blanks were utilized. Furthermore, as far as the utilized tool is regarded, it was made in H13 steel quenched at $1020{ }^{\circ} \mathrm{C}$, characterized by a 52 HRc hardness; a cylindrical pin was used (height $4 \mathrm{~mm}$, diameter $4 \mathrm{~mm}$ ); the tool penetration was $4.2 \mathrm{~mm}$, the matrix radii were equal to $3.5 \mathrm{~mm}$ and the tool nuting angle $(\theta)$ was equal to $3.5^{\circ}$ [4]. It should be observed that the both the matrix radius and the nuting angle strongly determines the effectiveness of the material flow during the FSW of $\mathrm{T}$-joints and in this way the performances of the obtained parts are definitively influenced by such values. As the technological parameters are regarded a tool rotating speed (R) of 1000 r.p.m. and a tool feed rates (Vf) of $150 \mathrm{~mm} / \mathrm{min}$ were utilized [4].

In order to investigate the material flow occurring during the FSW process $0.1 \mathrm{~mm}$ thick foils of commercially pure copper $(99.95 \%)$ were placed between the adjoining edges of the blanks to be welded, i.e. between the skin and the stringer: in this way during the welding process the stirring action of the tool pin distributes in the space the copper along specific flow directions. The subsequent investigation on the welded specimens was developed along the transverse section of the joint. The copper was chosen on the basis of its large melting temperature value (about $1083^{\circ} \mathrm{C}$ ), good ductility and different color with respect to the aluminum alloy. Several tests were developed and the sections taken from the specimens were analyzed by both micro and macro observations. In order to obtain such results the specimens were embedded by hot compression mounting, polished and finally etched with Keller reagent for $45 \mathrm{~s}$ and observed by a light microscope.

\subsection{Numerical model for $T$ joints}

The commercial FEA software DEFORM-3D ${ }^{\mathrm{TM}}$, Lagrangian implicit code designed for metal forming processes, has been utilized to model the FSW process. The workpiece was modelled as a rigid visco-plastic material; the welding tool and the clamping fixture fillet radii were assumed rigid, while the rest of the experimental clamping fixture was taken into account through proper boundary conditions. The FSW numerical simulation was divided into two stages: the sinking stage and the welding (advancing) one. During the sinking stage the tool moves down vertically at $0.1 \mathrm{~mm} / \mathrm{sec}$ with the prescribed rotating speed. Then, during welding or advancing stage, the rotating tool moves along the welding line. The sinking stage is modelled to reach high enough temperature for the subsequent welding process and the advancing stage is modelled to investigate the thermo-mechanical phenomena in the formation of the weld nugget. A rigid-viscoplastic temperature and strain rate dependent material model was employed. The tool, modelled as rigid body, was meshed, for the thermal analysis, with about 3,000 tetrahedral elements.

As far as the modelling of the workpiece is regarded, a "single block" continuum model (continuum $\mathrm{T}$ profile) is used in order to avoid contact instabilities due to the intermittent contact at the sheet-sheet and 
sheet-tool interfaces [6]. In Figure 2 the initial "sharp" $\mathrm{T}$ profile together with the rigid clamping fixture fillet are highlighted.

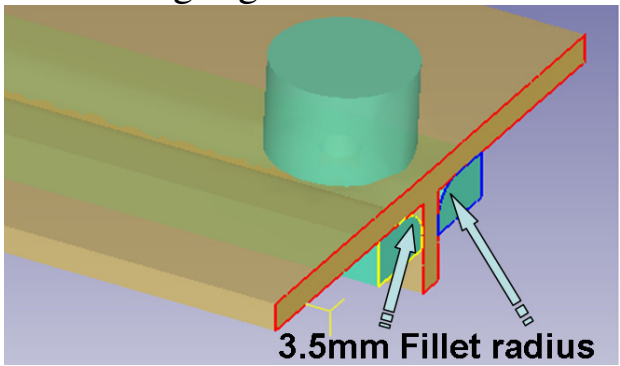

Fig. 2. Sketch of the utilized FEM model

The rotating tool moves forward and welds a crack left behind the pin as it advances along the welding line. The starting $\mathrm{T}$ profile, with blanks $3 \mathrm{~mm}$ in thickness, was meshed with about 12,000 tetrahedral elements with single edges of about $0.75 \mathrm{~mm}$; in this way about four element were placed along the sheet thickness. A non-uniform mesh with adaptive remeshing was adopted. In order to highlight the material flow due to the FSW processes, i.e. the nodes movements induced by the tool action, the point tracking option of the software DEFORM$3 \mathrm{D}^{\mathrm{TM}}$ was utilized, highlighting for a set of nodes initially placed along the welding line, their final position after deformation.

\section{OBTAINED RESULTS}

Figure 3 shows the transverse section of a welded joint. As mentioned before, the copper marker initially lays between the skin and the stringer, right at the interface between the two sheets; as a consequence of the tool pin action, the copper foil has been deformed and partially fractured.

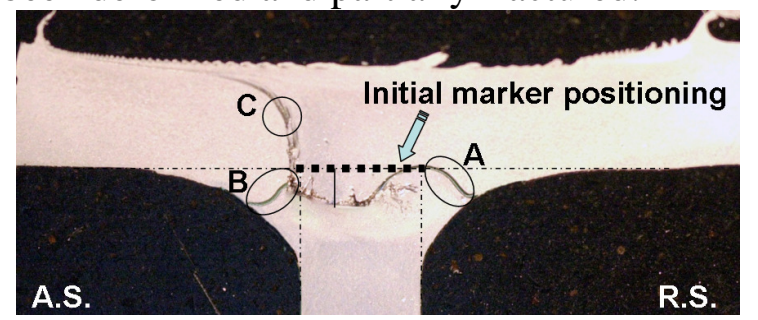

Fig. 3. Macro image of the etched transverse section of a welded joint

The etching process that the specimen has undergone to clearly highlights the final positioning of the copper foil. In particular it can be seen from the picture that the peripheral areas of the foil have been deformed and "pushed" down and out to the sides of the final joint, in the fillet zones (A and $\mathrm{B}$ marks in Fig.3). On the contrary, the central part of the foil has been fractured by the tool stirring action and "split" into two directions: downward, right under the pin, close to the symmetry axis of the joint; upward and to the left, i.e. toward the advancing side, in the skin (C mark in Fig.3).

As far as the A and B areas are regarded, the point tracking option of DEFORM-3D ${ }^{\mathrm{TM}}$ allowed analyzing, from numerical point of view, the final shape of the marker (Figure 4).

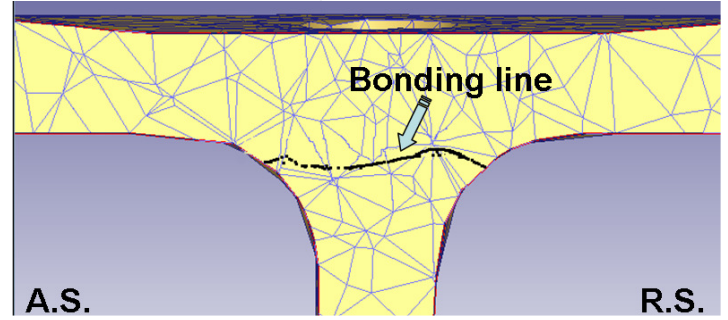

Fig. 4. FEM: tracked points final position (transverse section)

A path line made of 100 points initially laying at the interface between the two sheets was used, and the movement of such points was observed after the tool passed by the considered section. The above figure shows how the model is able to reproduce the "wavy" profile of the final marker positioning, highlighting the upward and downward components of the flux as well as the outward components that result in the formation of the fillets in the final joint through the forging action of the tool. In this way the actual bonding line is found both from a numerical and an experimental point of view. It is important to notice that a forward velocity component is found for the tracked point. This is highlighted by the fact that the tracked points are no more in a unique transverse section, as they were before the welding, but they are found all over a certain area along the tool advancing direction. Such aspect can be better understood looking at the next Figure 5.

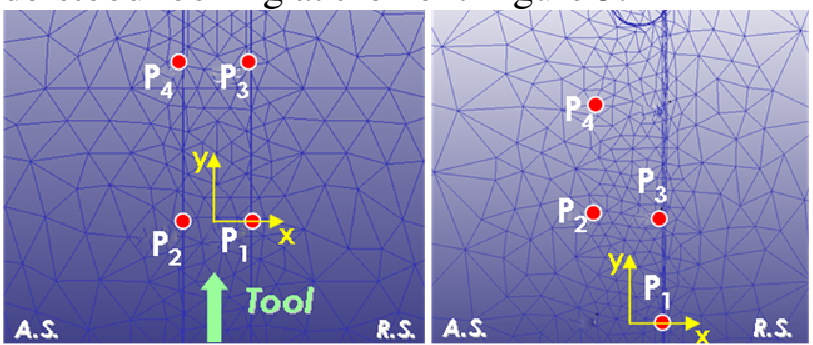

Fig. 5. FEM: tracked points initial and final position (horizontal section)

Four points, namely P1-P4, initially laying at the edges of the utilized marker in a horizontal section at the bottom of the skin, have been tracked. In detail, the two points in the retreating side rotate a few degrees with the tool and end at approximately the same y. The two points in the advancing side rotate 
with the tool for about $180^{\circ}$ being left far from their original $\mathrm{y}$ in the tool advancing direction. As far as the $\mathrm{C}$ area is regarded it has to be noticed that the final positioning of the marker found in such zone of the welded $\mathrm{T}$ joint section is very similar to the corresponding marker positioning found for a butt joint [10]: this indicates that, as for butt joints, also in $\mathrm{T}$ joint FSW processes there exists a material flow going from the retreating side to the advancing side, passing by the trailing edge of the weld. Figure 6 shows the numerical result obtained in terms of nodal velocity along the horizontal direction.

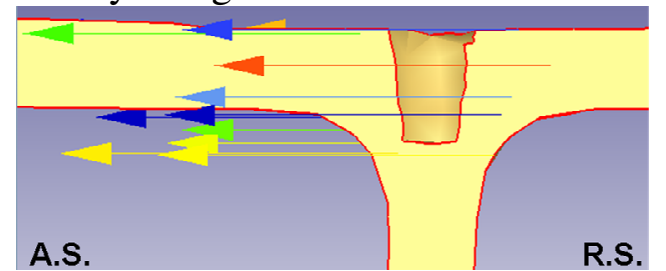

Fig. 6. FEM: horizontal component of the nodal velocity

As it can be seen from the above figure, referred to the trailing edge of the weld, when the crack opened by the tool pin is closing, the material flows to the advancing side, where part of the actual bonding line is found. It is important to notice that one main difference between FSW of T joints and butt joints is that in the former process the initial separation surface between the sheets to be welded is horizontal, while in the latter is vertical. In FSW of $\mathrm{T}$-joints a strong vertical component of the material flow is found, due to the reaction of the stringer material that does not participate into the bonding; in other words, the bottom part of the stringer behaves like a "wall", and the material pushed down by the tool action "bounces back" against it and comes upward to the skin. Such phenomenon is highlighted in the next Figure 7, referred to the same instant and location of Figure 6. In the picture it can be observed that a vertical component of nodal velocity is present in the advancing side of the weld, carrying some material, and part of the fragmented marker, in the skin.

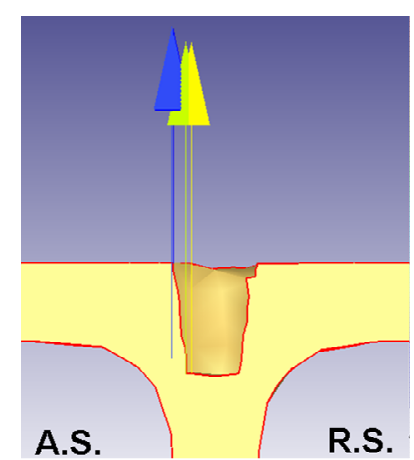

Fig. 7. FEM: vertical component of the nodal velocity

\section{CONCLUSIONS}

The material flow in the FSW of AA2024-T4 T joints has been investigated through numerical simulations and experimental tests, utilizing a thin foil of copper as marker. The following conclusions are drawn:

$\checkmark$ The actual bonding line is found under the initial separation between the skin and the stringer, due to a downward and outward component of the material flow.

$\checkmark$ In the skin the occurring flow is similar to the one observed in FSW of butt joints.

$\checkmark$ An upward flow is found resulting in a fragmented marker in the advancing side of the skin, approximately where the bonding line is found in butt joints.

The acquired information can be successfully utilized in order to chose effective set of process parameters, improving the process mechanics and avoiding the insurgence of defects.

\section{ACKNOWLEDGEMENTS}

This work has been performed with funding from MIUR (Italian Ministry for Instruction, University and Research).

\section{REFERENCES}

1. H.J. Liu, H. Fujii, M. Maeda, K. Nogi, Tensile properties and fracture locations of friction-stir-welded joints of 2017-T351 aluminium alloy, J. of Mat. Proc. Tech., 142 (2003) 692-696.

2. M. Guerra, C. Schmidt, L.C. McClure, L.E. Murr, A.C. Nunes, Flow patterns during friction stir welding, Materials characterization 49 (2003) 95-101.

3. K. Erbsloh, C. Dalle Donne, D. Lohwasser, Friction stir welding of T-joints, Materials Science Forum 426-432 (4), (2003) 2965-2970

4. L. Fratini, G. Buffa, L. Filice, F. Gagliardi, FSW of AA6082-T6 T-joints: process engineering and performance measurement, Proceedings of the Institutions of Mechanical Engineers, Part B, Journal of Engineering Manufacture, 220 (5), (2006) 669-676..

5. M. Guerra, C. Schmidt, L.C. McClure, L.E. Murr, A.C. Nunes, Flow patterns during friction stir welding, Materials characterization, 49 (2003) 95-101.

6. G. Buffa, J. Hua, R. Shivpuri, L. Fratini, A continuum based FEM model for friction stir welding - model development, Mat. Science and Eng. A, 419/1-2, (2006) 381-388.

7. L. Fratini, G. Buffa, D. Palmeri, J. Hua, R. Shivpuri, Material flow in FSW of AA7075-T6 butt joints: numerical simulations and experimental verifications, Science and Technology of Welding and Joining, Vol. 11, $\mathrm{n}^{\circ} 4$, (2006) 412-421. 